\title{
Three cases of didelphys uterus, including uterus didelphys with Obstructed Hemivagina and Ipsilateral Renal Agenesis (OHVIRA Syndrome) AKA herlyn-werner-wunderlich syndrome, with a systematic review
}

\begin{abstract}
Background: Herlyn-Werner-Wunderlich (HWW) syndrome also known as obstructed hemivagina ipsilateral renal agenesis (OHVIRA) is characterized by the presence of a didelphys uterus, obstructed hemivagina and ipsilateral renal agenesis. OHVIRA most commonly presents with symptoms of menarche pelvic pain, dysmenorrhea and an abdominal mass. Surgical intervention is often required to correct malformations and reduce the incidence of pelvic endometriosis, infection, and adhesions, and infertility for these patients. We present three cases MDA with various presentations and clinical courses.
\end{abstract}

Objective: Review of mullerian and other uterine abnormalities relationship to reproductive performance and pregnancy Outcome.

\section{Presentation of cases}

a. Case 1: 15 year old Hispanic female, G0P0, presented with Primary dysmenorrhea, mullerian anomaly and solitary kidney. Ultrasound and Magnetic Resonance Imaging (MRI) showed uterus didelphys with obstructed hemivagina and ipsilateral renal agenesis (OHVIRA Syndrome) AKA (Herlyn-Werner-Wunderlich Syndrome)

b. Case 2: A 35 years old Hispanic female, Gravida 2, Para 2002 with history of two previous cesarean deliveries presented with retained intrauterine device (IUD). The IUD strings could not be visualized on the exam but ultrasound and MRI revealed IUD in the right endocervical canal in the lower portion of the uterus. Diagnostic hysteroscopy confirmed the diagnosis of didelphys uterus with a horizontal vaginal septum. The retained IUD was clearly visible on the lower cervix under the vaginal septum and was easily removed with ring forceps.

c. Case 3: 24 year old Hispanic Female, G1P0, at 6 4/7 weeks gestational age, presented for her first prenatal visit. Bedside ultrasound showed second mass next to the uterus, official ultrasound revealed duplicated uterine horns and cervices. Differential diagnosis includes uterine didelphys and bicornuate bicollis uterus. Single live intrauterine pregnancy within the right uterine horn noted. Patient declined pelvic MRI for further evaluation of didelphys uterus despite counseling that MRI was safe imaging tool during pregnancy.

Discussion: Mullerian duct anomalies (MDA) encompass a large range of defects resulting from an abnormal fusion or failure of fusion of paramesonephric ducts during embryogenesis. Patient 1,2 and 3 were all diagnosed with didelphys uteri; However for each patient, the clinical symptomatology, diagnostic imaging and management was different. MDAs are present in a small percentage of the population. However, incidence increases among women with a history of recurrent pregnancy loss and many
Volume 7 Issue 2 - 2017

\author{
Shadi Rezai,' Celia Ligorski, ${ }^{2}$ Alexander \\ Hughes, ${ }^{3}$ Paul N Fuller,' Cassandra E \\ Henderson ${ }^{4}$ \\ 'Department of Obstetrics and Gynecology, Southern California \\ Kaiser Permanente, USA \\ ${ }^{2}$ Rowan University School of Osteopathic Medicine, USA \\ ${ }^{3}$ St. George's University, West Indies \\ ${ }^{4}$ Department of Obstetrics and Gynecology, Lincoln Medical \\ and Mental Health Center, USA
}

\begin{abstract}
Correspondence: Cassandra E Henderson, Pace University, School of Nursing, I Pace Plaza, New York, NY, I0038, USA, Email Cassandra.henderson@nychhc.org
\end{abstract}

Received: April 18, 2017 | Published: May 26, 2017

are incidental findings such as occurred in patients two and three. If ultrasound suggests MDA, it can be confirmed by magnetic resonance imaging (MRI), which is the gold standard for diagnosis. A new classification system for HWW was created in 2015 at the Peking Union Medical College Hospital by Zhu et al. ${ }^{2}$ Symptomatic treatment of these malformations can be hormonal or surgical. The patient in case 1 was placed on hormonal therapy to improve dysmenorrhea and pelvic pain. However, surgical intervention was ultimately needed to correct her anomaly thereby lowering the risk of infection, adhesions, endometriosis, miscarriages and preterm labor.

Conclusion: Mullerian duct anomalies encompass a range of uterine abnormalities that occur because of interrupted development and fusion of the paramesonephric ducts during embryogenesis. Diagnosis may occur as a consequence of clinical symptoms, fertility complications or as an incidental finding. Mullerian duct abnormalities of a specific uterine anomaly and renal findings characterize OHVIRA/HWW. Ultrasound and MRI play an important role in prompt diagnosis of MDAs and HWW often being discovered during prenatal care. Prompt diagnosis and treatment is needed to ensure relief of symptoms, prevention of infection and preservation of fertility.

Keywords: Congenital Mullerian Anomaly, Didelphys Uterus, Double Uterus, Dysmenorrhea, Hematocolpos, Herlyn-Werner-Wunderlich Syndrome, Horizontal Vaginal Septum, Intrauterine Device, OHVIRA Syndrome, Pregnancy Loss, Pregnancy Outcome, Preterm Labor, Primary Dysmenorrhea, Mullerian Duct Anomalies, Pregnancy, Prematurity, Renal agenesis, Reproductive Performance, Vaginal Septum, Retained IUD

Abbreviations: HWW, Herlyn Werner Wunderlich Syndrome; MRI, Magnetic Resonance Imaging; MDA, Mesonephric Duct Anomalies; MRI, Mullerian Anomalies; MDAs, Mullerian Duct Anomalies; RPL, Recurrent Pregnancy Loss; Retained Intrauterine Device ; Uterus didelphys; OHVIRA Syndrome, Uterus Didelphys With Obstructed Hemivagina And Ipsilateral Renal Agenesis

\section{Background}

Herlyn-Werner-Wunderlich (HWW) syndrome also known as obstructed Hemivagina Ipsilateral renal agenesis (OHVIRA) is characterized by the presence of a didelphys uterus, obstructed Hemivagina and Ipsilateral renal agenesis. ${ }^{1-3}$ It is a very rare genitourinary tract anomaly involving the Mullerian and Mesonephric 
duct development, most often occurring during the sixth to ninth week of embryologic development. ${ }^{4}$ In the general population, Mullerian duct anomalies (MDA) have a prevalence of $2-3 \%$, with $11 \%$ and $43 \%$ having uterus didelphys and renal abnormalities, respectively. ${ }^{5}$

OHVIRA most commonly presents during menarche as pelvic pain, dysmenorrhea and an abdominal mass. ${ }^{5-8}$ Depending on the level of embryologic malformation, management commonly involves surgical intervention in the form of excision of the vaginal septum or hemihysterectomy. ${ }^{1-5}$ In addition to relieving the pain and discomfort associated with the collection of debris and blood, surgical intervention may reduce the incidence of pelvic endometriosis, infection, adhesions and infertility. ${ }^{5-9}$ The significance of embryologic maldevelopment corresponds to the differences in clinical symptoms, management, and diagnosis. We present three cases MDA, one with renal agenesis, all with various presentations and clinical courses.

\section{Presentation of the case}

\section{Case I}

A 15-year-old Hispanic female, G0P0, initially presented with persistent sharp right lower quadrant pain. The patient was treated for pelvic inflammatory disease but, during further evaluation to rule out ovarian cyst and ovarian torsion, the patient was discovered to have severe primary dysmenorrhea, MDA and a solitary kidney. Ultrasound (Figure 1) and magnetic resonance imaging (MRI) (Figure 2) revealed a uterus didelphys with obstructed Hemivagina and Ipsilateral renal agenesis (OHVIRA syndrome) also known as Herlyn-Werner-Wunderlich Syndrome (Figure 3). Ultrasound indicated a $7.1 \times 7.0 \times 7.2 \mathrm{~cm}$ thick-walled right adnexal structure containing debris and fluids, which represented uterine didelphys with hematometra of the right-sided uterine horn (Figure $1 \& 2$ ). There was also a left uterine horn, which was oriented in the left pelvis and appeared unremarkable. Initial reproductive endocrinology and infertility recommendation was for 6 months of Leuprolide (Lupron) followed by continuous oral contraceptives (OCP) (with no placebo week), which the patient completed. However, the patient started getting her menstrual period every month (heavy and painful) while on OCP. The OCP was stopped and once the bleeding stopped the patient was re-started on 6 months of Lupron with norethindrone add back therapy, followed by continuous OCP (with no placebo week). The patient is currently scheduled to discuss the surgical option of hemi hysterectomy of the right rudimentary uterus versus resection of vaginal septum to open of obstructed Hemivagina. ${ }^{10}$
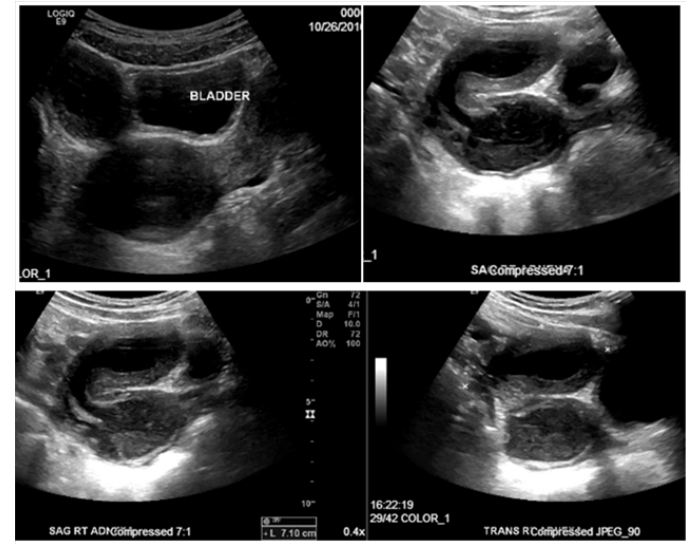

Figure I Patient I: Pelvic Ultrasound, Transverse View 10/26/16: A 7.I x 7.0 $\times 7.2 \mathrm{~cm}$ thick-walled right adnexal structure containing debris and fluid, this represent uterine didelphys with hematometra of the right-sided uterine horn. There is also uterine horn, which is oriented in the left pelvis and appears unremarkable.

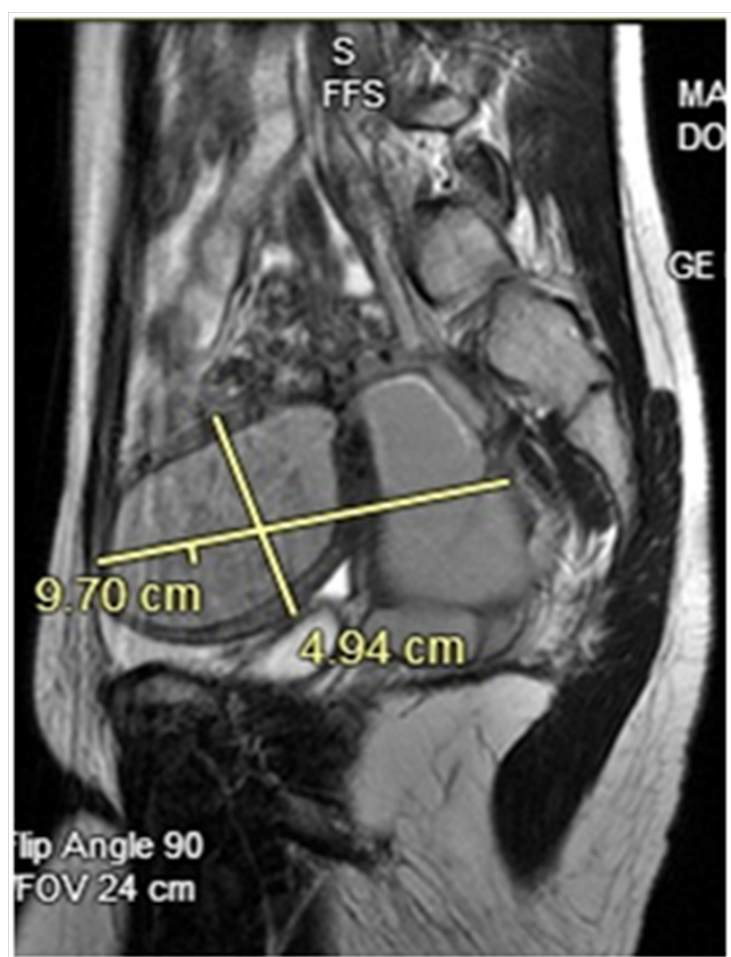

Figure 2 Patient I: Pelvic MRI:A normal-appearing uterine horn is seen in the left pelvis measuring $4.2 \times 1.7 \times 2.1 \mathrm{~cm}$. A $9.7 \times 4.9 \times 4.7 \mathrm{~cm}$ lobular tubular thick wall cystic structure in the right pelvis is seen. Signal intensity suggests hemorrhagic contents. This lesion is contiguous with a similar but smaller dilated tubular structure extending to the right aspect of the posterior vagina. Findings are suspicious for uterine didelphys with hematometra of the rightsided uterine horn and cervix.

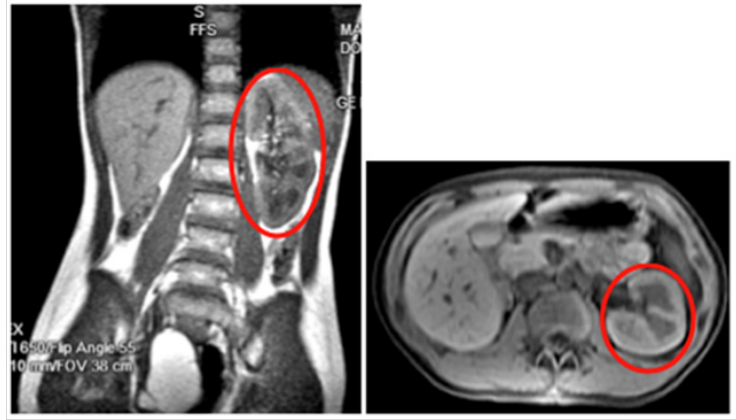

Figure 3 Patient I: MRI of Abdomen with and without Contrast: Showing normal left kidney (Red Circle) with Right Renal Agenesis.

\section{Case 2}

35 years old Hispanic female, G2P2002 with history of two previous cesarean deliveries presented with a retained intrauterine device (IUD). A Para Gard IUD was placed 4 years ago in Mexico and on exam the IUD string could not be visualized. Ultrasound (Figure 6) and MRI (Figure 7) revealed the IUD in the right end cervical canal in the lower portion of the one of her two uteruses. Diagnostic hysteroscopy confirmed the two uterine cavities were not connected to each other and therefore the diagnosis of two separate uteri (didelphys uterus) was confirmed. Further physical examination revealed the patient had a horizontal vaginal septum (Figure 5) with two separate cervices on each side of vaginal septum. Once the retained IUD was clearly visualized in the lower cervix hiding under the vaginal septum it was removed easily with ring forceps. 


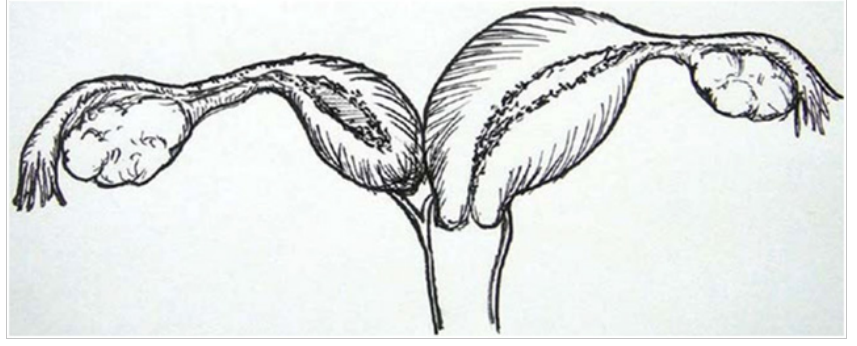

Figure 4 Patient I: Classification I.2, cervicovaginal atresia without communicating uteri: unremarkable left Uterus, cervix and vagina on the left, with non-communicating Right rudimentary horn of bicornuate uterus and cervicovaginal atresia on the right. ${ }^{2}$ Figure reprinted with permission from Wolters Kluwer Medknow Publications. ${ }^{2}$

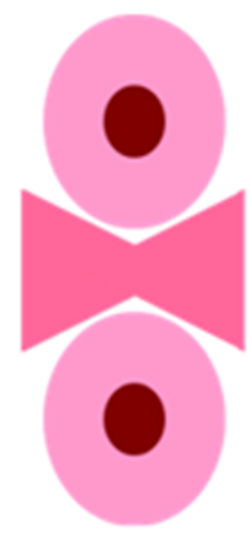

\section{Cervix}

\section{Cervical Canal} Vaginal Septum

Figure 5 Patient 2: Patient had horizontal vaginal septum as well as two separate cervices in each side of vaginal septum, and ParaGard IUD was in the lower cervix. ${ }^{31}$
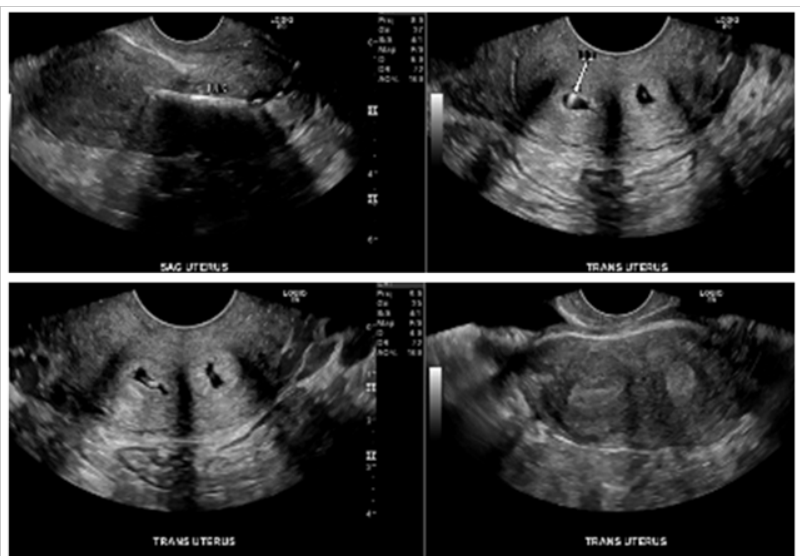

Figure 6 Patient 2: Pelvic Ultrasound: didelphys uterus with two endocervical canals. The IUD is seen in the right endocervical canal. Patient is still at risk for pregnancy via the patent left cervical canal.

\section{Case 3}

A 24-year-old Hispanic female, G1P0 at $64 / 7$ weeks gestational age, presented for her first prenatal visit. Bedside ultrasound showed a second mass next to the uterus and official ultrasound revealed duplicated uterine horns and cervices with a single live intrauterine pregnancy within the right uterine horn (Figure 9). Differential diagnosis includes uterine didelphys and bicornuate bicollis uterus. However, the patient declined pelvic MRI for further evaluation of didelphys uterus, despite counseling on the safety of MRI during pregnancy.

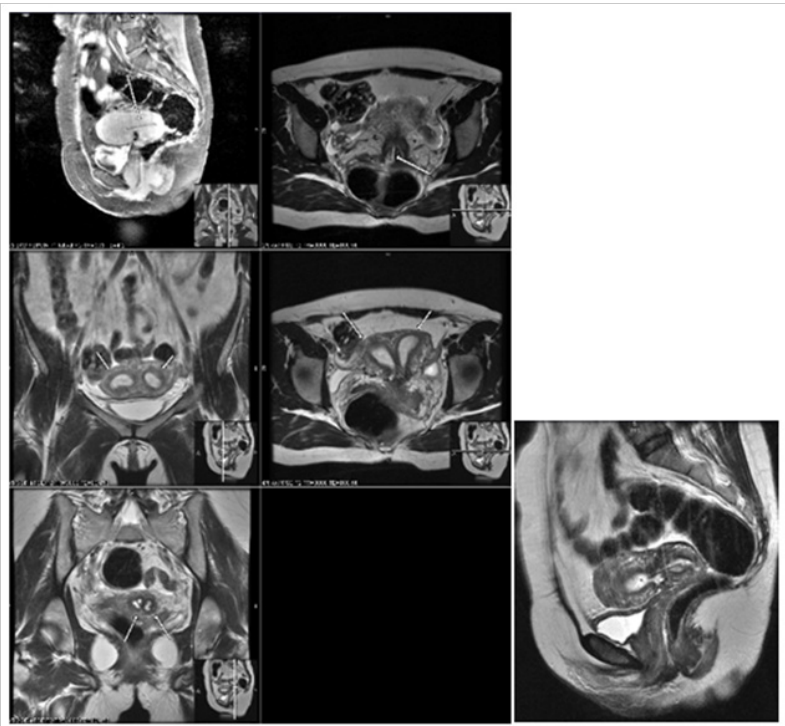

Figure 7 Patient 2: Pelvic MRI showing 2 uteri, 2 separate cervices/duplicated cervix. Intrauterine Device (IUD) within the right uterine horn is visualized.

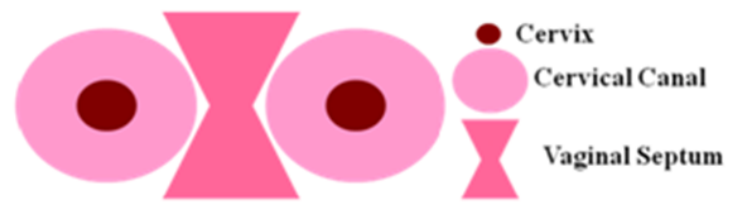

Figure 8 Patient 3: Patient hadVertical vaginal septum as well as two separate cervices in each side of vaginal septum, Pregnancy in the right uterine horn. ${ }^{20,21}$

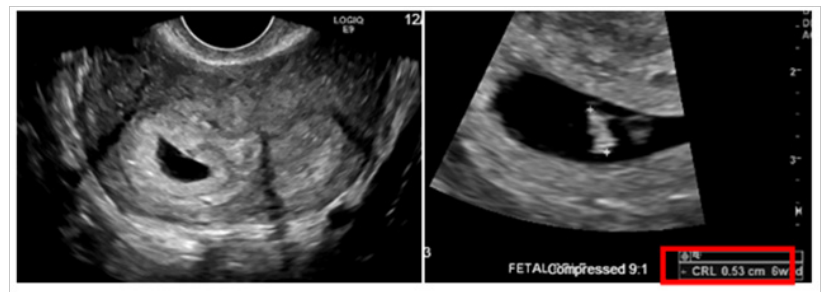

Figure 9 Patient 3: Pelvic Ultrasound: Differential diagnosis includes uterine didelphys and bicornuate bicollis uterus. Single live intrauterine pregnancy within the right uterine horn corresponding to a sonographic age of 6 weeks 2 days noted.

\section{Discussion}

Mullerian duct anomalies (MDA) encompass a large range of defects resulting from an abnormal fusion or failure of fusion of paramesonephric ducts during embryogenesis. ${ }^{4}$ Patients 1,2 and 3 were all diagnosed with didelphys uteri. However, clinical symptomatology, diagnostic imaging and management were different for each patient.

Their initial symptoms leading to a diagnosis of MDA vary widely. Although, MDAs are present in a small percentage of the population, the incidence increases for women who have experienced recurrent miscarriages ${ }^{11,12}$ MDAs are not normally diagnosed on pelvic examination, but may be discovered on ultrasound during evaluation of pregnancy or pelvic pain, as many are incidental findings such as occurred with patients 2 and $3 .^{11,13}$ The diagnosis of MDA through 
an incidental finding is not uncommon as a non-obstructed uterus maybe asymptomatic with normal menstruation often delaying the diagnosis..$^{2,14,15}$

A patient's presentation will depend on the degree of duct anomaly. The patient in case 1 presented with severe dysmenorrhea and bleeding from the non-obstructed uterine horn. In contrast, case 2 and 3 patients were asymptomatic with uterine anomalies incidental findings during routine ultrasound imaging. Definitive diagnoses were made in cases 2 and 3 of didelphic uteri while case 1 had the addition of ipsilateral renal agenesis further classifying it as OHVIRA syndrome. Case 1 illustrates why screening for renal abnormalities is important in patients with MDA as renal agenesis is the most commonly reported urologic anomaly associated with MDA (classifying it as OHVIRA). However renal duplication and multicystic dysplastic kidney have also been reported. ${ }^{16,17}$

Transvaginal ultrasound is commonly used as an initial step for investigating pelvic pain. If ultrasound suggests MDA, MRI can confirm it, which is the gold standard for diagnosis. ${ }^{1,18}$ Three dimensional ultrasounds have also shown promising results but, are not available in many clinical settings. ${ }^{13,19-21}$ Currently, no evidence exists demonstrating any harmful effects of MRI to the fetus and if clinically necessary should be encouraged, as in case $3 .^{22}$

Several classifications systems already exist for MDA. ${ }^{23,24}$ However, a new classification system for HWW was created in 2015 at the Peking Union Medical College Hospital by Zhu et al. ${ }^{2}$ This classification system is used to separate HWW patients into two classes based on the presence of obstruction. ${ }^{2}$ In class 1 , patients have a completely obstructed Hemivagina with either a patent proximal cervix (class 1.1) or a non patent cervix (class 1.2) (Figure 4). ${ }^{2}$ In class 2 there is incomplete obstruction and either communication through the vaginal septum (class 2.1) or through the uterus (class 2.2). ${ }^{2}$

In all three cases pelvic ultrasound was initial imaging tool. The ultrasound in case 1 (Figure 1) and subsequent MRI (Figure 2) shows a lobular tubular thick wall cystic structure in the right pelvis suggesting hemorrhagic contents extending into the right aspect of the posterior vagina and didelphys uterus. MRI of the abdomen depicts renal agenesis, demonstrating abnormal kidney development confirming HWW. As per the classification presented by Zhu and imaging, the patient has a class 1.2 OHVIRA. ${ }^{2}$ The ultrasounds in cases 2 and 3 (Figures $6 \& 9$ respectively) both demonstrate didelphys uterus with two cervices. The additional MRI in case 2, revealed two separate cervices and two uteri one with a retained IUD. MDA can be associated with a vaginal septum, more commonly vertical (Case 2 \& Figure 5) but cases of a horizontal septum have also been reported (Case 1 \& Figure 8$).{ }^{14}$

Symptomatic treatment of these malformations can be hormonal or surgical. Menstrual suppression with OCPs or Lupron can alleviate dysmenorrhea, decrease blood loss and improve quality of life. ${ }^{19,25}$ Surgical intervention can be used to remove obstruction or correct anatomy to prevent future complications such as miscarriages. ${ }^{26-28}$ The patient in case 1 was placed on hormonal therapy to improve dysmenorrhea and pelvic pain however; surgical intervention may ultimately be needed to correct her anomaly. ${ }^{2}$

\section{Conclusion}

Mullerian duct anomalies encompass a range of uterine abnormalities that occur because of interrupted development and fusion of the paramesonephric ducts during embryogenesis. Diagnosis may occur based on clinical symptoms, fertility complications or as an incidental finding. Mullerian duct abnormalities encompass a specific uterine anomaly and renal findings characterize OHVIRA/HWW. Ultrasound and MRI play an important role in prompt diagnosis of MDAs and HWW often being discovered during prenatal care. Even though $80 \%$ of women with MDA have successful implantation of an embryo into the uterine lining they are commonly associated with obstetrical complications such as recurrent spontaneous abortion. ${ }^{8,29,30}$ Therefore, prompt diagnosis and treatment is needed to ensure relief of symptoms, prevention of infection and preservation of fertility.

\section{Acknowledgments}

The authors would like to thank Ms. Judith Wilkinson, Medical Librarian at Lincoln Medical and Mental Health Center Science Library for providing the reference articles.

\section{Conflicts of interest}

None.

\section{References}

1. Piccinini PS, Doski J. Herlyn-Werner-Wunderlich syndrome: a case report. Rev Bras Ginecol Obstet. 2015;37(4):192-196.

2. Zhu L, Chen N, Tong JL, et al. New classification of Herlyn-WernerWunderlich syndrome. Chin Med J (Engl). 2015;128(2):222-225.

3. Smith NA, Laufer MA. Obstructed Hemivagina and Ipsilateral renal anomaly (OHVIRA) syndrome: management and follow-up. Fertility and Sterility. 2007;87(4):918-922.

4. Sadler TW, Langman J. Langman's Medical Embryology. Wolters Kluwer Health/Lippincott Williams \& Wilkins, Philadelphia, USA. 2015

5. Khaladkar SM, Kamal V, Kamal A, et al. The Herlyn-WernerWunderlich Syndrome - A Case Report with Radiological Review. Pol J Radiol. 2016;81:395-400.

6. Bajaj SK, Misra R, Thukral BB, et al. OHVIRA: Uterus didelphys, blind hemivagina and ipsilateral renal agenesis: Advantage MRI. J Hum Reprod Sci. 2012;5(1):67-70.

7. Stanislavsky A. Herlyn-Werner-Wunderlich syndrome (HWW). Radiopaedia. 2015.

8. Aveiro AC, Miranda V, Cabral AJ, et al. Herlyn-Werner-Wunderlich syndrome: a rare cause of pelvic pain in adolescent girls. BMJ Case Rep. 2011;p.1-3.

9. Kirkham YA, Ornstein MP, Aggarwal A, et al. Menstrual suppression in special circumstances. J Obstet Gynaecol Can. 2014;36(10):915-926.

10. Jones III HW, Rock JA. TeLinde's Operative Gynecology. Gynecologic Surgical Procedures. 1942;pp.578-579.

11. Nahum GG. Uterine anomalies, induction of labor, and uterine rupture. Obstet Gynecol. 2005;106(5 Pt 2):1150-1152.

12. Acien P. Reproductive performance of women with uterine malformations. Hum Reprod. 1993;8(1):122-126.

13. Christiansen ME, Detti L. Clinically Relevant Female Genital Tract Anomalies. Clin Obstet Gynecol. 2017;60(1):18-26.

14. Rezai S, Bisram P, Lora Alcantara I, et al. Didelphys Uterus: A Case Report and Review of the Literature. Case Reports in Obstetrics and Gynecology. 2015;2015(2015):1-5.

15. Saxena N, Channawar S, Khadkikar R, et al. HVIRA syndrome. JPGO. 2014;1(12).

16. Gungor UF, Bastu E, Gulsen G, et al. OHVIRA syndrome presenting with acute abdomen: a case report and review of the literature. Clin Imaging. 2014;38(3):357-359. 
17. Singh K, Thakur S, Soni A, et al. Herlyn-Werner-Wunderlich Syndrome /OHVIRA Syndrome; A Rare Urogenital Anomaly with Unusual Presentation in Two Cases with Review of Literature. Clinics Mother Child Health. 2016;13(1):1-4.

18. Takagi H, Matsunami K, Noda K, et al. Magnetic resonance imaging in the evaluating of double uterus and associated urinary tract anomalies: a report of five cases. J Obstet Gynaecol. 2003;23(5):525-527.

19. Raga F, Bonilla MF, Blanes J, et al. Congenital Mullerian anomalies: diagnostic accuracy of three-dimensional ultrasound. Fertil Steril. 1996;65(3):523-528.

20. Bermejo C, Cantarero R, Diaz D, et al. Three-dimensional Ultrasonography in the Diagnosis of Mullerian Duct Anomalies. Donald School Journal of Ultrasound in Obstetrics and Gynecology. 2009;3(1):21-30.

21. Chandler TM, Machan LS, Cooperberg PL, et al. Mullerian duct anomalies: from diagnosis to intervention. $\mathrm{Br} J$ Radiol. 2009;82(2009):1034-1042.

22. Bulas D, Egloff A. Benefits and risks of MRI in pregnancy. Semin Perinatol. 2013;37(5):301-304.

23. Buttram VC, Gibbons WE. Mullerian anomalies: a proposed classification. (An analysis of 144 cases). Fertil Steril. 1997;32(1):40 46.
24. The American Fertility Society. The American Fertility Society classifications of adnexal adhesions, distal tubal occlusion, tubal occlusion secondary to tubal ligation, tubal pregnancies, müllerian anomalies and intrauterine adhesions. Fertil Steril. 1988;49(6):944-955.

25. Lupron with Norethindrone Add-back Therapy.

26. Grimbizis GF, Camus M, Tarlatzis BC, et al. Clinical implications of uterine malformation and hysteroscopic treatment results. Hum Reprod Update. 2001;7(2):161-174.

27. Raga F, Bauset C, Remohi J, et al. Reproductive impact of congenital Mullerian anomalies. Hum Reprod. 1997;12(10):2277-2281.

28. Ludmir J, Samuels P, Brooks S, et al. Pregnancy outcome of patients with uncorrected uterine anomalies managed in a high-risk obstetric setting. Obstet Gynecol. 1990;75(6):906-910.

29. Sugiura OM, Ozaki Y, Suzumori N. Mullerian anomalies and recurrent miscarriage. Curr Opin Obstet Gynecol. 2013;25(4):293-298.

30. Tong J, Zhu L, Lang J. Clinical characteristics of 70 patients with Herlyn-Werner-Wunderlich syndrome. Int $J$ Gynaecol Obstet. 2013;121(2):173-175

31. Ludwin A, Ludwin I, Pitynski K, et al. Differentiating between a double cervix or cervical duplication and a complete septate uterus with longitudinal vaginal septum. Taiwan J Obstet Gynecol. 2013;52(2):308310 